Supplement of Biogeosciences, 17, 3367-3383, 2020

https://doi.org/10.5194/bg-17-3367-2020-supplement

(C) Author(s) 2020. This work is distributed under

the Creative Commons Attribution 4.0 License.

(c) (1)

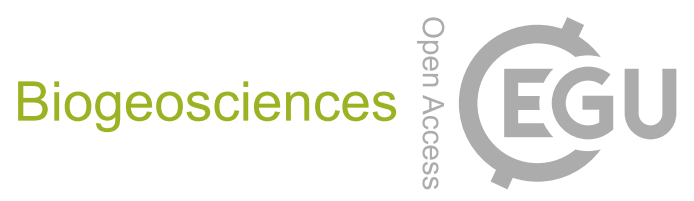

Supplement of

\title{
From fibrous plant residues to mineral-associated organic carbon - the fate of organic matter in Arctic permafrost soils
}

Isabel Prater et al.

Correspondence to: Isabel Prater (i.prater@tum.de)

The copyright of individual parts of the supplement might differ from the CC BY 4.0 License. 
Table S1: Basic properties of bulk soil samples and result of the fractionation: $\mathrm{pH}$ (measured in $\left.\mathrm{H}_{2} \mathrm{O}\right), \mathrm{EC}\left(\mu \mathrm{S} \mathrm{cm}^{-1}\right)$, bulk density $\left(\mathrm{g} \mathrm{cm}^{-3}\right), \mathrm{C}\left(\mathrm{mg} \mathrm{g}^{-1}\right), \mathrm{N}\left(\mathrm{mg} \mathrm{g}^{-1}\right), \mathrm{C} / \mathrm{N}$ ratio and the distribution of SOM fractions ( $\mathrm{mg} \mathrm{g}^{-1}$ ) for the bulk soil samples of all analyzed depth layers. The particulate organic matter fractions are free (fPOM), occluded (oPOM) and small occluded (oPOMs) particulate organic matter.

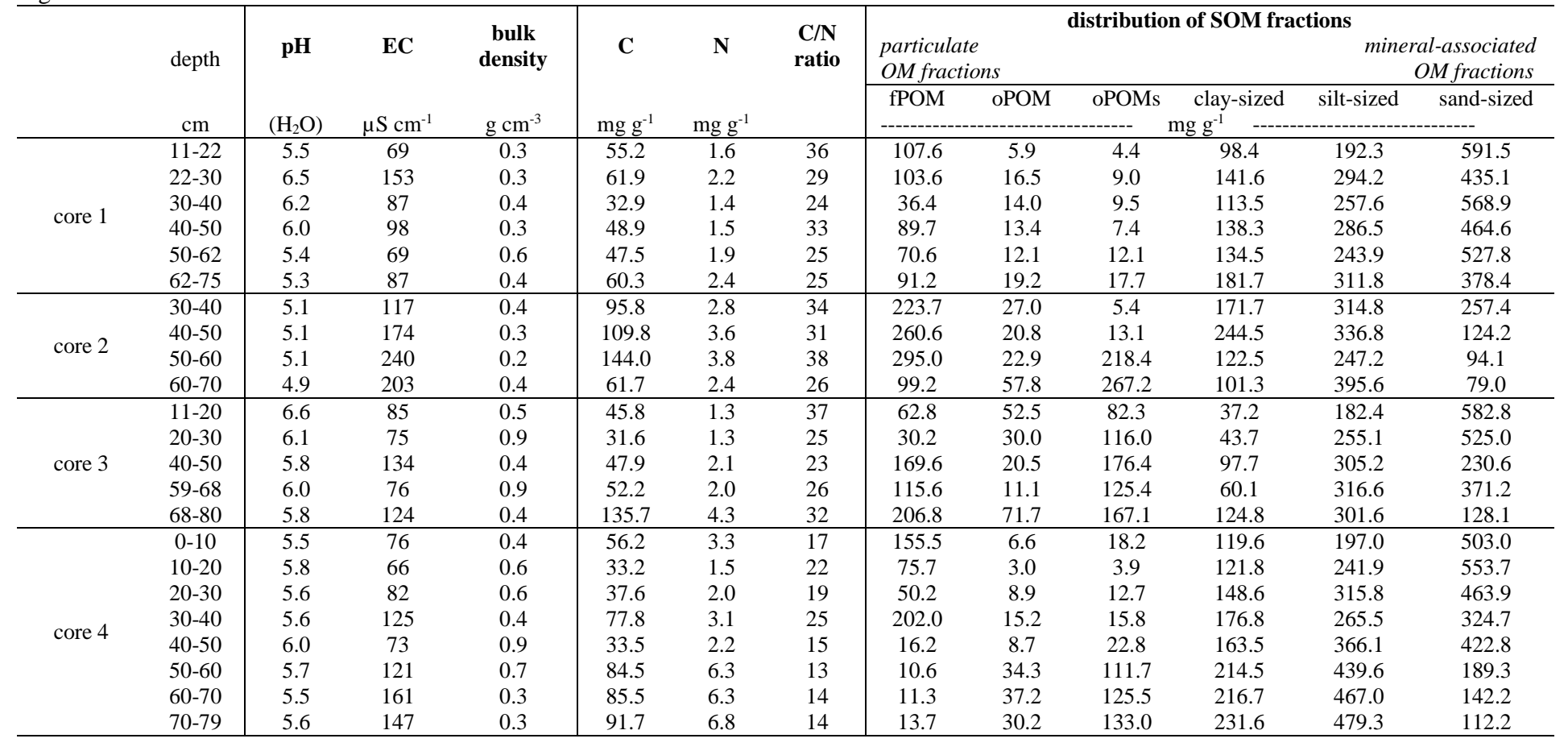


Table S2: Properties of SOM fractions: C per fraction $\left(\mathrm{mg} \mathrm{C}(\mathrm{g} \text { soil })^{-1}\right), \mathrm{N}$ per fraction $\left(\mathrm{mg} \mathrm{N}(\mathrm{g} \mathrm{soil})^{-1}\right), \mathrm{C} / \mathrm{N}$ ratio, $\delta^{13} \mathrm{C}\left(\%\right.$ V-PDB) and $\delta^{15} \mathrm{~N}\left(\%{ }_{0}\right.$ air $\left.\mathrm{N}_{2}\right)$ for free particulate (fPOM), occluded particulate (oPOM), small occluded particulate (oPOMs) and clay-sized mineral-associated organic matter (MAOM).

\begin{tabular}{|c|c|c|c|c|c|c|c|c|c|c|c|c|c|c|c|c|c|c|c|c|c|}
\hline \multirow{2}{*}{ 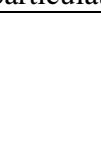 } & \multirow{2}{*}{$\begin{array}{c}\text { depth } \\
\mathrm{cm}\end{array}$} & \multicolumn{5}{|c|}{ fPOM } & \multicolumn{5}{|c|}{ oPOM } & \multicolumn{5}{|c|}{ oPOMs } & \multicolumn{5}{|c|}{ clay-sized MAOM } \\
\hline & & $\begin{array}{c}\text { C per } \\
\text { fraction } \\
\mathrm{mg} \mathrm{C} \\
{\text { (g soil })^{-1}}\end{array}$ & $\begin{array}{l}\text { N per } \\
\text { fraction } \\
\mathrm{mg} \mathrm{N} \\
(\mathrm{g} \mathrm{soil})^{-1}\end{array}$ & $\begin{array}{l}\mathrm{C} / \mathrm{N} \\
\text { ratio }\end{array}$ & $\begin{array}{c}\delta^{13} \mathrm{C} \\
\% \\
\text { V-PDB } \\
\end{array}$ & $\begin{array}{c}\delta^{15} \mathrm{~N} \\
\% \\
\text { air } \mathrm{N}_{2}\end{array}$ & $\begin{array}{l}\text { C per } \\
\text { fraction } \\
\mathrm{mg} \mathrm{C} \\
(\mathrm{g} \text { soil })^{-1}\end{array}$ & 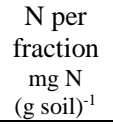 & $\begin{array}{l}\mathrm{C} / \mathrm{N} \\
\text { ratio }\end{array}$ & $\begin{array}{c}\delta^{13} \mathrm{C} \\
\% 0 \\
\text { V-PDB } \\
\end{array}$ & $\begin{array}{c}\delta^{15} \mathrm{~N} \\
\% \\
\text { air } \mathrm{N}_{2}\end{array}$ & $\begin{array}{l}\text { C per } \\
\text { fraction } \\
\mathrm{mg} \mathrm{C} \\
(\mathrm{g} \mathrm{soil})^{-1}\end{array}$ & $\begin{array}{l}\mathrm{N} \text { per } \\
\text { fraction } \\
\mathrm{mg} \mathrm{N} \\
(\mathrm{g} \mathrm{soil})^{-1}\end{array}$ & $\begin{array}{l}\mathrm{C} / \mathrm{N} \\
\text { ratio }\end{array}$ & $\begin{array}{c}\delta^{13} \mathrm{C} \\
\% \\
\text { V-PDB } \\
\end{array}$ & $\begin{array}{c}\delta^{15} \mathrm{~N} \\
\% \\
\text { air } \mathrm{N}_{2}\end{array}$ & 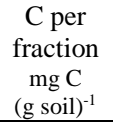 & $\begin{array}{l}\mathrm{N} \text { per } \\
\text { fraction } \\
\mathrm{mg} \mathrm{N} \\
(\mathrm{g} \mathrm{soil})^{-1}\end{array}$ & $\begin{array}{l}\mathrm{C} / \mathrm{N} \\
\text { ratio }\end{array}$ & $\begin{array}{c}\delta^{13} \mathrm{C} \\
\% \\
\text { V-PDB } \\
\end{array}$ & $\begin{array}{c}\delta^{15} \mathrm{~N} \\
\% \\
\text { air } \mathrm{N}_{2} \\
\end{array}$ \\
\hline \multirow{6}{*}{ core 1} & $11-22$ & 765.9 & 361.4 & 76 & -26.9 & 1.4 & 41.2 & 28.6 & 52 & -26.2 & 1.3 & 19.4 & 45.6 & 15 & -27.3 & 1.0 & 147.2 & 453.4 & 12 & -27.0 & 1.8 \\
\hline & $22-30$ & 641.6 & 366.3 & 51 & -28.2 & 0.7 & 108.3 & 56.4 & 55 & -27.8 & 1.0 & 36.0 & 61.2 & 17 & -28.0 & 0.9 & 188.8 & 428.7 & 13 & -28.1 & 1.8 \\
\hline & $30-40$ & 437.1 & 164.1 & 64 & -26.5 & 0.9 & 174.1 & 61.9 & 68 & -26.4 & 0.9 & 69.7 & 106.8 & 16 & -27.5 & 0.8 & 270.3 & 534.2 & 12 & -27.2 & 1.7 \\
\hline & $40-50$ & 674.8 & 311.1 & 71 & -26.8 & 0.9 & 106.7 & 51.5 & 67 & -25.8 & 1.2 & 27.6 & 54.1 & 17 & -27.1 & 1.0 & 152.2 & 445.9 & 11 & -27.2 & 2.1 \\
\hline & $50-62$ & 588.3 & 305.4 & 49 & -28.3 & 0.8 & 105.6 & 42.2 & 64 & -28.0 & 1.4 & 50.5 & 90.6 & 14 & -28.4 & 1.1 & 222.2 & 470.2 & 12 & -28.5 & -0.4 \\
\hline & $62-75$ & 533.1 & 300.3 & 44 & -26.0 & 1.0 & 134.6 & 56.1 & 60 & -25.3 & 1.0 & 76.7 & 114.4 & 17 & -26.9 & 1.1 & 218.4 & 438.0 & 13 & -26.9 & 1.1 \\
\hline \multirow{4}{*}{ core 2} & $30-40$ & $\begin{array}{l}780.3 \\
\end{array}$ & $\begin{array}{l}508.2 \\
\end{array}$ & 53 & -26.8 & 0.8 & & & outlier & & & 13.7 & 31.5 & 15 & \begin{tabular}{|l|}
-27.3 \\
\end{tabular} & 0.9 & 118.0 & 358.2 & 11 & \begin{tabular}{|l|}
-27.8 \\
\end{tabular} & 1.6 \\
\hline & $40-50$ & 722.0 & 438.5 & 50 & -27.1 & 0.9 & 71.7 & 35.0 & 63 & -26.5 & 1.1 & 30.7 & 55.5 & 17 & -26.9 & 0.7 & 158.2 & 424.3 & 11 & -27.5 & 1.6 \\
\hline & $50-60$ & 674.6 & 384.9 & 66 & -26.3 & 1.0 & 58.5 & 40.1 & 55 & -26.4 & 1.1 & 194.7 & 367.6 & 20 & -26.5 & 1.8 & 64.4 & 176.0 & 14 & -26.0 & 3.2 \\
\hline & $60-70$ & 538.8 & 226.8 & 61 & -25.7 & 0.7 & & & outlier & & & 265.7 & 514.4 & 13 & -26.6 & 2.8 & 92.7 & 161.5 & 15 & -26.9 & 3.4 \\
\hline \multirow{5}{*}{ core 3} & $11-20$ & 527.0 & 356.4 & 51 & -27.3 & -0.3 & & & outlier & & & 222.9 & 341.7 & 22 & -26.0 & 1.8 & 76.7 & 167.7 & 16 & -24.1 & 2.9 \\
\hline & $20-30$ & 373.9 & 229.3 & 41 & -26.9 & 0.8 & & & outlier & & & 394.5 & 469.5 & 21 & -25.8 & 2.3 & 99.1 & 175.8 & 14 & -25.5 & 3.0 \\
\hline & $40-50$ & & & outlier & & & 165.4 & 33.3 & 113 & -25.3 & 0.7 & 427.7 & 387.5 & 25 & -25.0 & 2.4 & 131.3 & 194.7 & 15 & -25.1 & 2.3 \\
\hline & $59-68$ & 608.3 & 463.1 & 35 & -25.8 & 0.7 & 82.1 & 33.9 & 64 & -26.2 & 0.7 & 216.3 & 293.9 & 19 & -25.6 & 2.9 & 59.4 & 118.5 & 13 & -25.5 & 3.1 \\
\hline & $68-80$ & 499.3 & 283.3 & 56 & -25.6 & 1.0 & 195.8 & 142.7 & 43 & -25.7 & 1.3 & 228.1 & 386.5 & 19 & -25.6 & 1.3 & 65.1 & 147.5 & 14 & -25.4 & 2.3 \\
\hline \multirow{8}{*}{ core 4} & $0-10$ & 543.0 & 385.7 & 24 & -27.9 & 0.4 & 50.6 & 23.7 & 37 & -26.9 & 1.5 & 105.7 & 141.3 & 13 & -28.2 & 0.2 & 250.7 & 371.2 & 12 & -27.7 & 0.3 \\
\hline & $10-20$ & 645.9 & 329.3 & 43 & -27.3 & 1.2 & 34.5 & 18.2 & 42 & -27.0 & 2.4 & 21.4 & 27.0 & 18 & -27.7 & 1.3 & 249.3 & 480.9 & 12 & -28.0 & 1.4 \\
\hline & $20-30$ & 419.3 & 199.1 & 39 & -30.2 & 1.3 & 102.1 & 54.2 & 35 & -30.4 & 2.4 & 94.3 & 108.4 & 16 & -30.1 & 1.5 & 311.7 & 506.3 & 11 & -30.4 & 1.6 \\
\hline & $30-40$ & 616.2 & 390.3 & 39 & -30.6 & 0.2 & 81.9 & 35.1 & 58 & -30.4 & 0.7 & 63.0 & 87.9 & 18 & -30.0 & 0.6 & 208.3 & 416.6 & 13 & -29.7 & 1.0 \\
\hline & $40-50$ & 157.0 & 85.6 & 28 & -30.1 & -0.2 & 114.3 & 60.0 & 29 & -30.6 & 0.2 & 224.7 & 202.4 & 17 & -30.6 & 0.3 & 431.4 & 522.9 & 12 & -31.2 & 0.6 \\
\hline & $50-60$ & 52.5 & 27.6 & 25 & -30.2 & 0.3 & 178.3 & 127.6 & 19 & -30.1 & 0.6 & 456.1 & 466.9 & 13 & -31.1 & 0.1 & 275.0 & 319.1 & 12 & -31.3 & 0.3 \\
\hline & $60-70$ & 55.1 & 34.3 & 22 & -31.2 & 0.0 & 190.1 & 140.4 & 18 & -30.5 & 0.6 & 473.8 & 481.6 & 13 & -31.5 & -0.0 & 223.7 & 263.7 & 12 & -31.8 & 0.4 \\
\hline & 70-79 & 63.6 & 39.6 & 22 & -30.4 & 0.5 & 147.9 & 104.3 & 19 & -30.0 & 0.7 & 479.7 & 485.8 & 13 & -31.2 & 0.2 & 249.2 & 289.96 & 12 & -31.6 & 0.2 \\
\hline
\end{tabular}


Table S3: Decomposition proxies a/oa ratio ${ }^{1}$ and 70-75/52-57 ratio $^{2}$ obtained by ${ }^{13} \mathrm{C}$ CP-MAS NMR spectroscopy per depth layer for free particulate (fPOM), occluded particulate (oPOM), small occluded particulate (oPOMs) and clay-sized mineral-associated organic matter (MAOM).

\begin{tabular}{|c|c|c|c|c|c|c|c|c|c|}
\hline \multirow{2}{*}{\multicolumn{2}{|c|}{$\begin{array}{c}\text { depth } \\
\mathrm{cm}\end{array}$}} & \multicolumn{2}{|r|}{ fPOM } & \multicolumn{2}{|r|}{ OPOM } & \multicolumn{2}{|r|}{ oPOMs } & \multicolumn{2}{|c|}{ clay-sized MAOM } \\
\hline & & $\mathrm{a} /$ oa ratio & 70-75/52-57 ratio & $\mathrm{a} / \mathrm{oa}$ ratio & 70-75/52-57 ratio & $\mathrm{a} /$ oa ratio & 70-75/52-57 ratio & a/oa ratio & 70-75/52-57 ratio \\
\hline \multirow{6}{*}{ core 1} & $11-22$ & 0.15 & 9.71 & 0.16 & 9.40 & 0.59 & 2.55 & 0.47 & 2.07 \\
\hline & $22-30$ & 0.15 & 6.96 & 0.16 & 8.68 & 0.58 & 2.49 & 0.52 & 2.26 \\
\hline & $30-40$ & 0.13 & 7.95 & 0.09 & 14.95 & 0.57 & 2.56 & 0.46 & 2.33 \\
\hline & $40-50$ & 0.12 & 11.38 & 0.12 & 10.27 & 0.63 & 2.23 & 0.62 & 2.06 \\
\hline & $50-62$ & 0.18 & 5.90 & 0.12 & 12.39 & 0.61 & 2.44 & 0.49 & 2.58 \\
\hline & $62-75$ & 0.21 & 5.21 & 0.10 & 11.57 & 0.61 & 3.15 & 0.47 & 2.53 \\
\hline \multirow{4}{*}{ core 2} & $30-40$ & 0.18 & 5.80 & \multicolumn{2}{|r|}{ outlier } & 0.54 & 2.68 & $n d$ & $n d$ \\
\hline & $40-50$ & 0.17 & 5.87 & 0.10 & 8.79 & 0.47 & 2.81 & $n d$ & $n d$ \\
\hline & $50-60$ & 0.12 & 7.36 & 0.14 & 5.68 & 0.34 & 2.77 & nd & nd \\
\hline & $60-70$ & 0.16 & 4.87 & \multicolumn{2}{|r|}{ outlier } & 0.39 & 1.83 & $n d$ & nd \\
\hline \multirow{5}{*}{ core 3} & $11-20$ & 0.14 & 5.64 & \multirow{2}{*}{\multicolumn{2}{|c|}{$\begin{array}{l}\text { outlier } \\
\text { outlier }\end{array}$}} & 0.25 & 3.07 & $n d$ & $n d$ \\
\hline & $20-30$ & 0.16 & 4.21 & & & 0.26 & 2.65 & $n d$ & $n d$ \\
\hline & $40-50$ & \multicolumn{2}{|r|}{ outlier } & 0.08 & 8.58 & 0.19 & 3.20 & 0.40 & 1.88 \\
\hline & $59-68$ & 0.25 & 3.80 & 0.09 & 5.81 & 0.28 & 2.77 & $n d$ & $n d$ \\
\hline & $68-80$ & 0.13 & 5.46 & 0.16 & 4.02 & 0.38 & 2.80 & $n d$ & $n d$ \\
\hline \multirow{8}{*}{ core 4} & $0-10$ & 0.21 & 4.18 & 0.22 & 6.48 & 0.50 & 2.70 & 0.43 & 2.17 \\
\hline & $10-20$ & 0.08 & 5.27 & 0.21 & 4.28 & 0.67 & 2.42 & 0.47 & 1.71 \\
\hline & $20-30$ & 0.11 & 5.17 & 0.30 & 4.36 & 0.68 & 2.27 & $n d$ & $n d$ \\
\hline & $30-40$ & 0.16 & 4.88 & 0.09 & 9.38 & 0.48 & 3.15 & nd & $n d$ \\
\hline & $40-50$ & 0.34 & 3.16 & 0.29 & 4.64 & 0.75 & 2.12 & 0.53 & 1.85 \\
\hline & $50-60$ & 0.32 & 3.75 & 0.39 & 3.79 & 0.55 & 2.50 & $n d$ & $n d$ \\
\hline & $60-70$ & 0.57 & 2.21 & 0.41 & 3.63 & 0.58 & 2.52 & $n d$ & $n d$ \\
\hline & 70-79 & 0.38 & 4.34 & 0.42 & 3.83 & $0 . .55$ & 2.65 & $n d$ & $n d$ \\
\hline
\end{tabular}

${ }^{1}$ Ratio of alkyl C and O/N alkyl C according to Baldock et al. (1997).

${ }^{2}$ Ratio of the chemical shift regions 70 to 75 ppm and 52 to $57 \mathrm{ppm}$ according to Bonanomi et al. (2013). 
Figure S1: Exemplary ${ }^{13} \mathrm{C}$ CP-MAS NMR spectra of free particulate OM (fPOM), occluded particulate OM (oPOM), small occluded particulate OM (oPOMs) and clay-sized mineral-associated OM (MAOM) fraction of one depth layer.

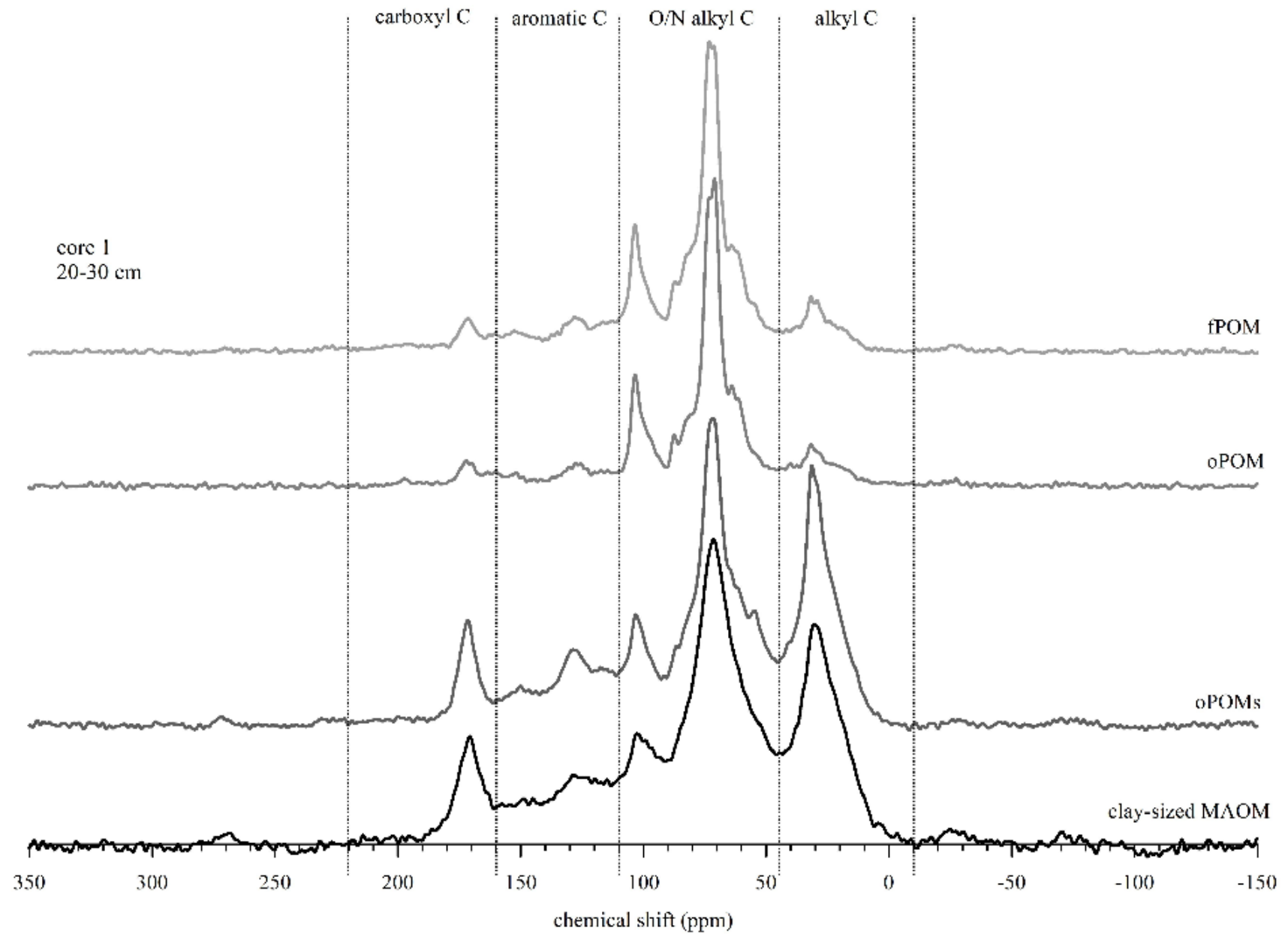

